\title{
A Novel Proximity-Based Range-Free Positioning Algorithm for Wireless Sensor Network Nodes
}

\author{
https://doi.org/10.3991/ijoe.v14i10.9308 \\ Huaimeng Gui \\ Shaanxi Polytechnic Institute, Xianyang, China \\ 304913013@qq.com
}

\begin{abstract}
This paper aims to reduce the estimated distance error (EDE) and location error (LE) of hop-based range-free node positioning algorithms. For this purpose, a novel range free positioning algorithm was proposed based on proximity, denoted as PNN-MAP. First, the proximity was introduced to express the distance between neighbouring nodes and the proximity expression was derived based on the geometric relationship between these nodes. Then, the estimated distance was calculated by the proximity expression and relevant information of anchor nodes, and the unknown node positions were estimated by the MDS-MAP to reduce the LE. Later, the PNN-MAP algorithm was simulated and compared to three other node positioning algorithms. The results show that the proposed algorithm achieved the smallest EDE and LE at different communication radiuses or different number of anchor nodes. In terms of energy consumption, the PNNMAP had a relatively high traffic volume. However, there is no requirement on the calculation ability of network nodes, and the estimated node positions can be displayed directly on the server after the positioning. In general, the PNN-MAP enjoys a strong practicality and universality. This research promotes the application of wireless sensor networks (WSNs).
\end{abstract}

Keywords-Wireless Sensor Network (WSN), Estimated Distance Error (EDE), Location Error (LE), Range-Free, Proximity

\section{Introduction}

The wireless sensor network (WSN) is able to monitor the environmental information in the area of the test node, such as temperature, humidity, pressure, speed and illuminance. However, the monitoring would be meaningless without knowing the positions of these data. In various applications (e.g. military tactical communication and target tracking), the information acquired by each sensor node must be bound to the node position [1-3].

The WSN is almost inaccessible to personnel, owing to the huge number and airdrop deployment of network nodes. Thus, the node positions cannot be determined in advance but positioned by a certain method. Currently, the popular positioning methods include global positioning system (GPS) and manual deployment [4-6]. Among them, the GPS is the most commonly used approach. Nevertheless, the GPS module may 
increase the cost, energy consumption and size of wireless sensors. Node positioning has become a key bottleneck of WSN applications, under such constraints as sensor node configuration, energy, calculation, storage and communication abilities. To solve the bottleneck, the positioning of WSN nodes must carry the following features: selforganization, low energy consumption, high fault-tolerance and scalability.

Fruitful results have been achieved on multi-nodal positioning, including rangebased positioning methods and range-free positioning methods. Specifically, the rangebased positioning methods rely on physical ranging techniques like angle of arrival (AOA), time of arrival (TOA), time difference of arrival (TDOA) and received signal strength indicator (RSSI). This type of strategy requires a strict environment and additional ranging hardware. By contrast, the range-free positioning methods mainly calculate the node positions based on the connectivity between nodes, such as four-corner distance vector-hop (DV-HOP), distance vector-regulated neighbourhood distance (DV-RND) and localized encryption and authentication protocol (LEAP). These three range-free positioning algorithms are respectively based on anchor deployment, distance vector routing and single-hop correction value [7-9].

Considering the strict environment and equipment requirements of range-based methods, this paper adopts the range-free methods to analyse the positioning of WSN nodes. First, the proximity parameter was obtained through the calculation of the distance relationship between neighbouring nodes. Based on the proximity, the distance between neighbouring nodes was derived by ranging positioning. Then, the actual positions of unknown nodes were evaluated by the two-phase positioning method based on greedy algorithm.

\section{Proximity-Based Range-Free Positioning}

The current range-free positioning algorithms generally estimate node distance based on the hop count. Most of them suffer from a huge positioning error. To solve the problem, a proximity-based range-free positioning algorithm was proposed: the probabilistic neural network-maximum a posteriori (PNN-MAP) algorithm. By this algorithm, the node distance is evaluated based on node information and proximity estimation, and then the node positions are obtained by multidimensional scaling-maximum a posteriori (MDS-MAP) algorithm [10-12].

\section{$2.1 \quad$ Network node}

Suppose there is an n-node WSN whose nodes are randomly deployed, belong to the same type and share the same communication radius $r$. Let $m$ be the number of anchor nodes, and $i$ and $j$ be two random nodes. Assuming that each neighbouring node obtains its information through the transmission and forwarding by node i, the following relationship can be established:

$\mathrm{M}_{i}=\left\{j \mid j \neq i \& \& d_{i j} \leq r\right\}$ 
where $\mathrm{M}_{i}$ is the set of neighbouring nodes of node $\mathrm{i} ; d_{i j}$ is the distance between node $\mathrm{i}$ and node $\mathrm{j}$.

\subsection{Proximity PNN}

Proximity refers to the distance between two neighbouring nodes [13-15]. Figure 1 shows the communication coverages of neighbouring nodes $i$ and $j$. Note that the communication coverage of each node is expressed as a circle with radius $r$.
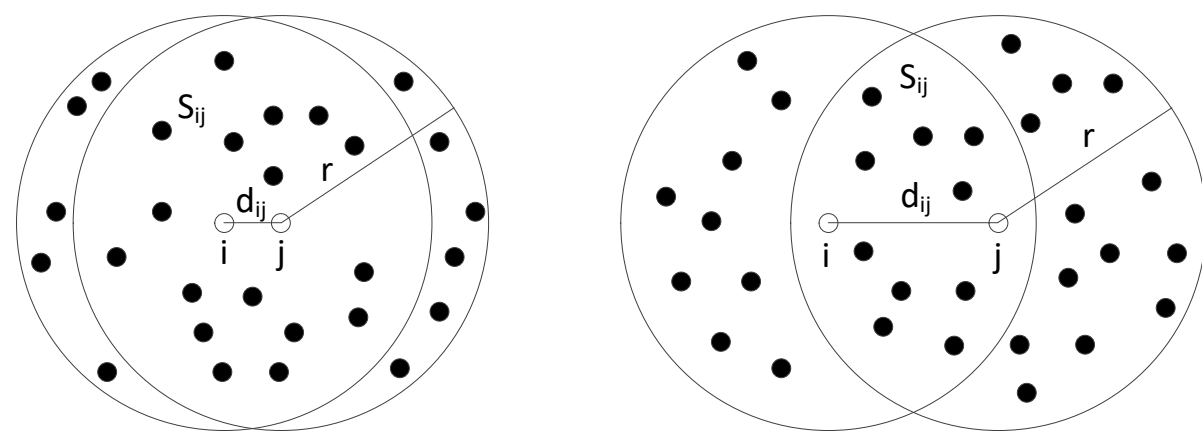

Fig. 1. Communication coverages of neighbouring nodes

As shown in the figure, $S_{i j}$ is the overlap between the communication coverages of neighbouring nodes $i$ and $j$; the black dots are the other neighbouring nodes of the two nodes. Then, the area of $S_{i j}$ can be expressed as:

$\mathrm{A}\left(\mathrm{S}_{i j}\right)=2 \mathrm{r}^{2} \arccos \left(\frac{d_{i j}}{2 r}\right)-d_{i j} \sqrt{r^{2}-\frac{d_{i j}^{2}}{4}}$

The, the ratio of this area to the communication radius of each node can be expressed as:

$\frac{A\left(S_{i j}\right)}{\pi r^{2}}=1-\frac{2}{\pi}\left(\frac{d_{i j}}{r}\right)+\frac{1}{12 \pi}\left(\frac{d_{i j}}{r}\right)^{3}+\frac{1}{320 \pi}\left(\frac{d_{i j}}{r}\right)^{5}+\cdots$

where $\frac{d_{i j}}{r}$ falls in the range of $[0,1]$. Thus, the terms starting from $\frac{1}{12 \pi}\left(\frac{d_{i j}}{r}\right)^{3}$ are negligible. Then, the two numerical values of the above equation are linearly correlated. Figure 2 illustrate the results of equation (3) after ignoring the negligible terms. 


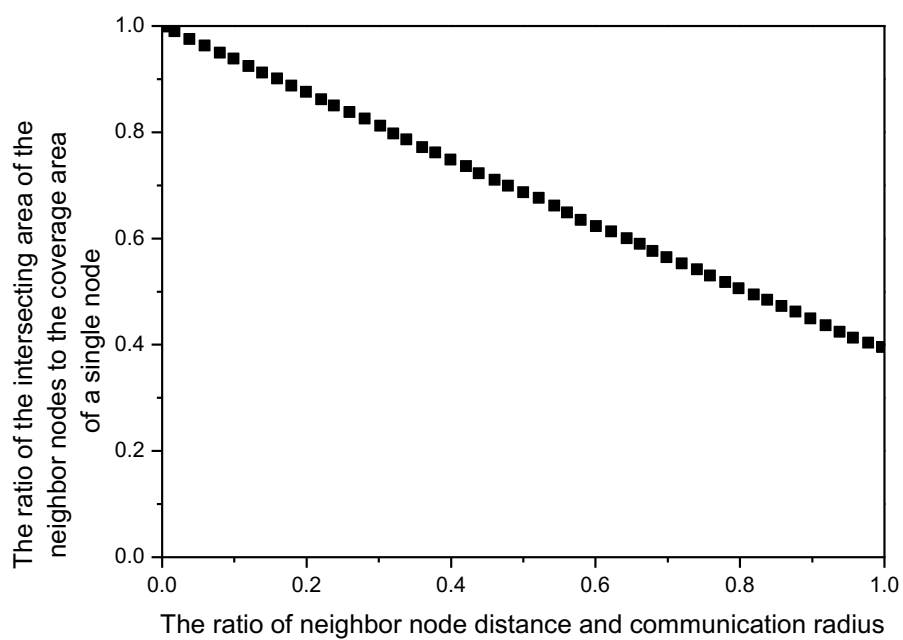

Fig. 2. The ratio of neighbouring node distance to communication radius the ratio of distance to the ratio of area value

As shown in Figure 2, the curve is approximately linear. Based on the values in that figure, we have the following linear expression:

$\frac{d_{i j}}{r}=\frac{1}{0.609}\left(1-\frac{A\left(s_{i j}\right)}{\pi r^{2}}\right)$

According to the Monte-Carlo statistical simulation method, the overlap area and communication coverage can be evaluated as:

$\frac{A\left(s_{i j}\right)}{\pi r^{2}} \approx \sigma \cdot \frac{N_{i j}}{N_{i}}$

where $N_{i j}$ is the number of nodes; $N_{i}$ is the number of nodes in the communication coverage of node $\mathrm{i} ; \sigma$ is the correction coefficient to minimize the error.

Assuming that there are sufficient neighbouring nodes around nodes $i$ and $j$, the proximity $P N N_{i j}$ can be obtained from equations (4) and (5):

$P N N_{i j}=P N N_{j i}=\frac{d_{i j}}{r}=\frac{1}{0.609}\left(1-\sigma \cdot \frac{N_{i j}}{\max \left(N_{i}, N_{j}\right)}\right)$

\subsection{PNN-MAP positioning algorithm}

According to equation (6), the distance between neighbouring nodes $i$ and $j$ can be estimated as:

$\overline{d_{\imath \jmath}}=\overline{d_{\jmath l}}=r * P N N_{i j}$ 
The estimated distance may have some error due to the elimination of the negligible terms. To reduce the error, the proximity correction factor $\alpha_{P N N}$ was introduced. Then, the distance between the neighbouring node can be finalized as $\alpha_{P N N} * P N N_{i j}$. Based on this final distance, the positions of the unknown nodes were estimated by the MDSMAP algorithm. This approach can be called the PNN-MAP algorithm.

\section{$3 \quad$ Simulation and Analysis}

To validate the proposed proximity-based range-free algorithm (PNN-MAP), ten WSNs were randomly constructed. Each WSN is $100 \mathrm{~m} * 100 \mathrm{~m}$ in size and contains 200 random nodes. All nodes are of the same type and able to communicate effectively. Then, these networks were simulated on Matlab to see how the PNN-MAP performs at different number of anchor nodes and communication radiuses. Besides, the performance of the PNN-MAP was contrasted with that of the DV-HOP, LEAP and DVRND. Below is a brief introduction to the simulation results.

\subsection{Performance comparison parameter}

The positioning performance of WSNs is mainly measured by energy consumption and positioning error. When the node distance is estimated by proximity, an error may occur in the estimation of distance. Therefore, the estimated distance error (EDE) was introduced to compare the performance of the said algorithms:

$\mathrm{EDE}=\frac{1}{r \sum_{i=1}^{n}\left|M_{i}\right|} \sum_{i=1}^{n} \sum_{j \in M_{i}}\left|d_{i j}-\bar{d}_{i j}\right| \times 100 \%, \mathrm{~d}_{i j}=\sqrt{\left(x_{i}-x_{j}\right)^{2}+\left(y_{i}-y_{j}\right)^{2}}$

where $\left(x_{i}, y_{i}\right)$ and $\left(x_{j}, y_{j}\right)$ are the actual coordinates of nodes $\mathrm{i}$ and $\mathrm{j}$, respectively; $d_{i j}$ and $\bar{d}_{i j}$ are the actual distance and estimated distance between nodes $i$ and $\mathrm{j}$, respectively; $\left|M_{i}\right|$ is the number of neighbouring nodes of node i.

The unknown node positions are estimated by maximum likelihood estimation in DV-HOP, LEAP and DV-RND algorithms, and MDS-MAP in the PNN-MAP algorithm. The location error (LE) can be expressed as:

$\mathrm{LE}=\frac{1}{(n-m) r} \sum_{i=1}^{n-m} \sqrt{\left(x_{i}-\bar{x}_{j}\right)+\left(y_{i}-\bar{y}_{j}\right)} \times 100 \%$

\subsection{Correction coefficient $\sigma$}

The correction coefficient $\sigma$ was introduced to reduce the error generated when the area ratio is replaced by the number of nodes. The most suitable value of $\sigma$ was determined by measuring the EDEs at different correction coefficients. The measured results are recorded in Figure 3, where the number of anchor nodes is 10, the range of $\sigma$ is $[0.5$, $1]$ and the communication radiuses are $15 \mathrm{~m}, 20 \mathrm{~m}$ and $25 \mathrm{~m}$. 


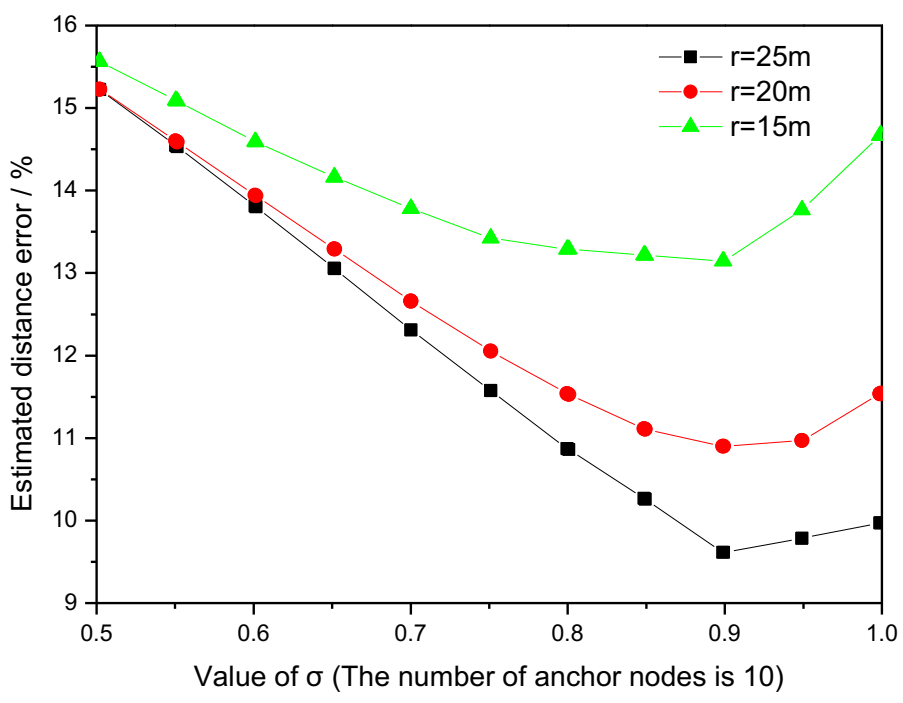

Fig. 3. Effect of the value of $\sigma$ on the EDE

As shown in Figure 3, the EDE firstly declined and then increased with the growth in the value of $\sigma$. Besides, the EDE is negatively correlated with the communication radius, i.e. the communication coverage. Whichever the communication radius, the EDE was minimized at the $\sigma=0.9$. Hence, the optimal value of the correction coefficient is 0.9 .

\subsection{Effect of communication radius on EDE and LE}

For a sensor node, the number of neighbouring nodes increases with the communication radius, i.e. the communication coverage. Therefore, the effectiveness of node positioning algorithms can be tested by changing the communication radius.

The WSN has a poor connectivity if the communication radius is too small, while each node consumes too much energy if the radius is too large. Considering this, the effect of communication radius on algorithm performance was tested with 20 anchor nodes and the communication radius of $[13,30]$ (unit: $\mathrm{m}$ ). Figures 4 and 5 respectively present the EDEs and LEs of the contrastive algorithms at different communication radiuses.

It can be seen from Figure 4 that the PNN-MAP achieved the smallest EDE, followed in ascending order by DV-RND, DV-HOP and LEAP. With the growth of the communication radius, the EDE of DV-HOP slightly increased from $20.0 \%$ to $21.3 \%$, while that of LEAP first decreased and then increased in the range of $[21.0 \%, 22.5 \%]$. The EDE ranges of both algorithms were small, a signal of weak dependence on communication radius. On the contrary, the EDEs of DV-RND and PNN-MAP plunged deeply with the increase of the communication radius. The EDE ranges of these two algorithms were $[19.3 \%, 11.9 \%]$ and $[15.2 \%, 9.2 \%]$, respectively. These results show that the PNN-MAP algorithm boast the smallest error and the highest effect. 


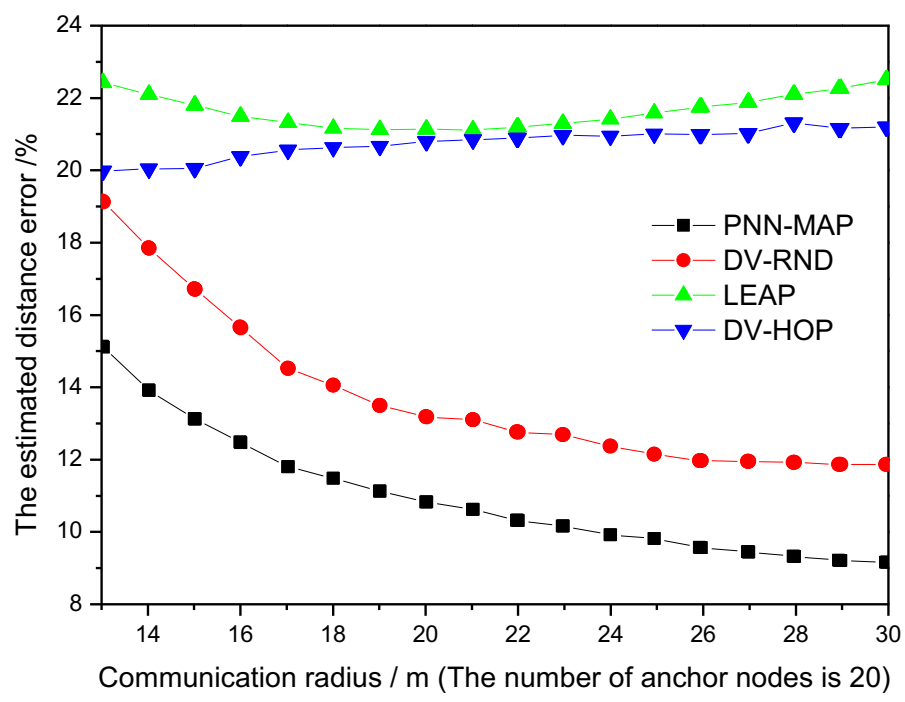

Fig. 4. Effect of communication radius on the EDE

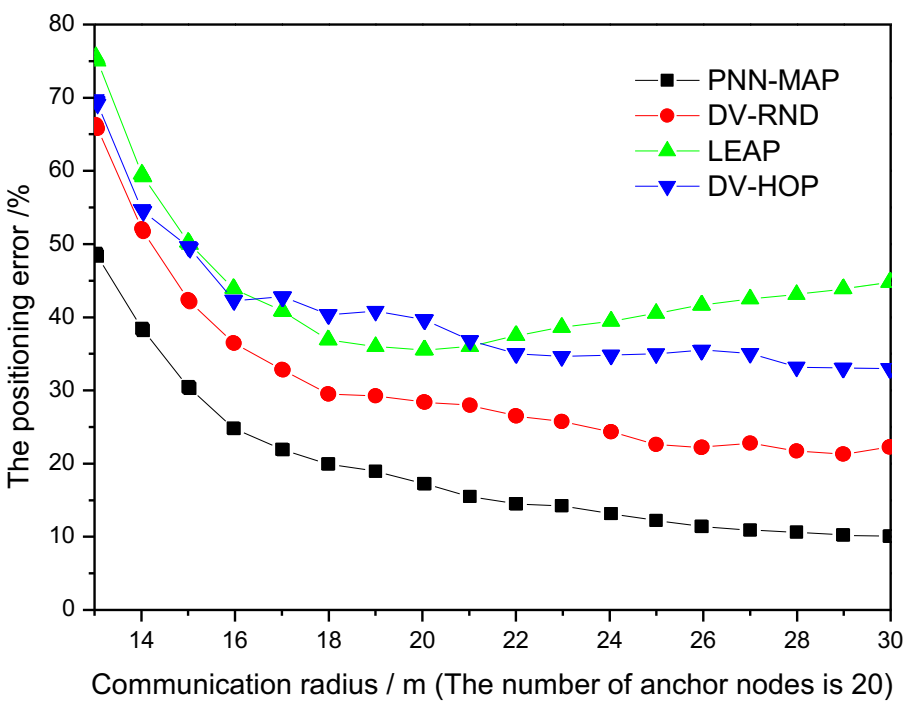

Fig. 5. Effect of communication radius on the LE

Since the proximity was introduced to accurately locate the actual position of each node, the effect of communication radius on the LE was further tested. The test results are displayed in Figure 5. It can be seen from this figure that the PNN-MAP algorithm had the smallest LE, followed by DV-RND. With the increase of the communication radius, obvious decline occurred in the LEs of PNN-MAP, DV-RND and DVHOP. The most significant decline belongs to PNN-MAP. The LE of LEAP decreased first and increased later. Overall, the PNN-MAP enjoys the best node positioning effect. 


\subsection{Effect of the number of anchor nodes on EDE and LE}

The impact of the number of anchor nodes on EDE and LE should also be investigated, for these nodes are the basis for node distance estimation and positioning calculation. Thus, the communication radius was set to $25 \mathrm{~m}$ and the range for the number of anchor nodes to $[3,20]$. This is because the positioning of a plane needs at least 3 nodes, but too many nodes may lead to high energy consumption and high cost. Figures 6 and 7 respectively show the test results on the effect of the number of anchor nodes on EDE and LE.

As shown in Figure 6, with the increase in the number of anchor nodes, the EDE of DV-HOP first decreased and then remained stable, while that of PNN-MAP, DV-RND and LEAP maintained at the same level, that is, the number of anchor nodes has no impact on the EDE at the same communication radius. This phenomenon can be explained as follows: the single-hop correction value in the LEAP is only related to the communication radius; the single-hop correction value in the DV-HOP algorithm, the RND correction value in the DV-RND algorithm, and the PNN correction value in the PNN-MAP algorithm are adjusted based on the node distance, but the corresponding EDE changes very slighting with the variation of the number of anchor nodes. In addition, it can be seen from Figure 6 that the PNN-MAP yielded the smallest EDE (9.8\%), followed by DV-RND (12.2\%). By contrast, the EDEs of LEAP and DV-HOP were both above $20 \%$. Hence, the PNN-MAP outperforms the other three algorithms in distance estimation.

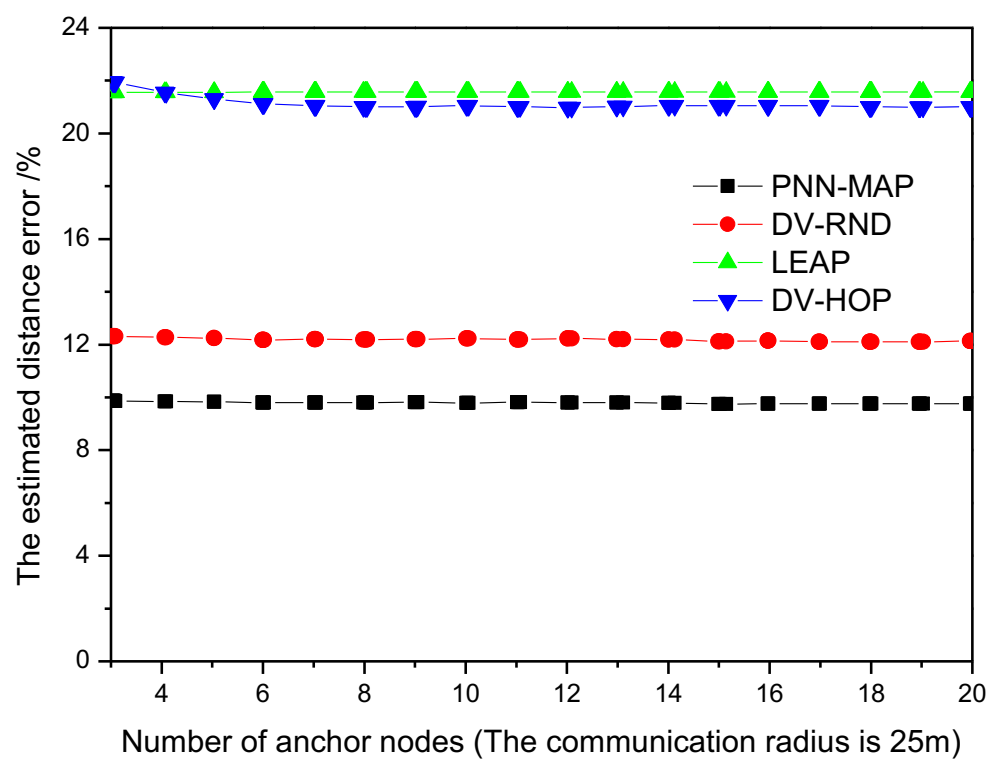

Fig. 6. Effect of the number of anchor nodes on the EDE 


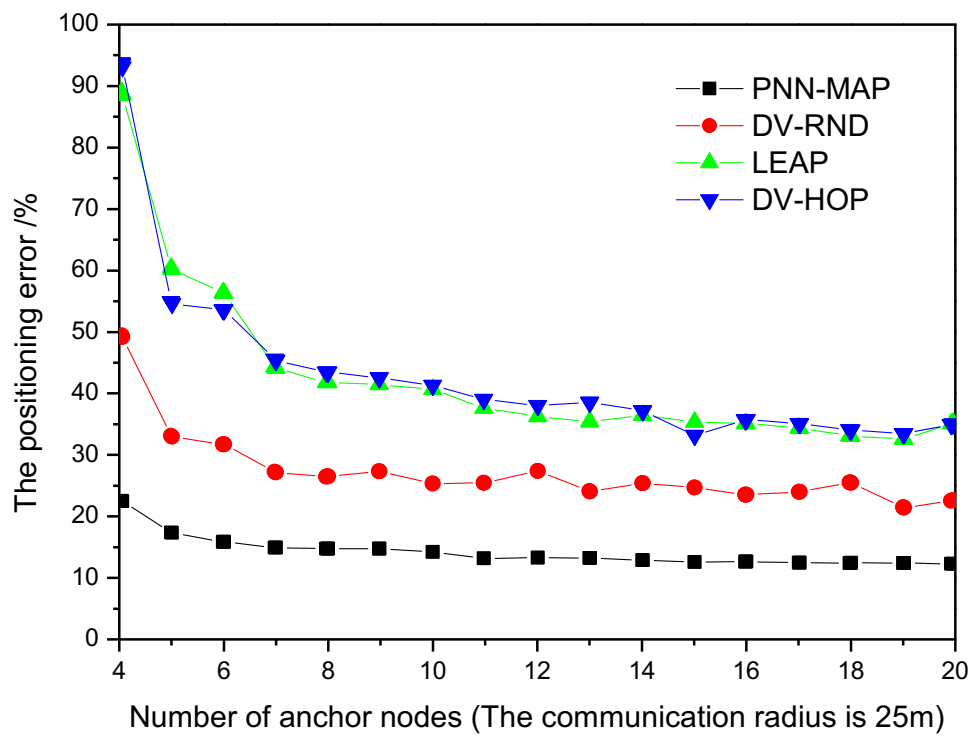

Fig. 7. Effect of the number of anchor nodes on the LE

All the algorithms outputted a high LE under three anchor nodes, indicating that the data is unreliable in this case. Thus, the number of anchor nodes was set to the range of $[4,20]$ to test its influence on the LE. According to Figure 7 , the LE of each algorithm exhibited a decreasing trend with the addition of anchor nodes. Besides, DV-HOP had a very similar LE with the LEAP, the PNN-MAP realized the smallest LE, and the DVRND achieved the second smallest LE. These results demonstrate the advantage of the PNN-MAP in node positioning at the fixed communication radius.

\subsection{Energy consumption analysis}

Under the energy constraint, lots of energy is consumed by WSN nodes during the positioning. To save energy in this process, it is necessary to compare the energy consumption of different algorithms. The energy consumption can be measured by the traffic volume under the same network conditions. The traffic volumes of the said four algorithms are listed in Table 1.

Table 1. Traffic volumes of different algorithms

\begin{tabular}{|l|l|}
\hline \multicolumn{1}{|c|}{ Name } & \multicolumn{1}{c|}{ Traffic volume } \\
\hline PNN-MAP & $\begin{array}{l}\text { Each neighbouring node obtains its information through the transmission and forwarding } \\
\text { by node i, and then transfer its information to the server. }\end{array}$ \\
\hline DV-HOP & Each anchor node broadcasts two messages to the entire network. \\
\hline LEAP & $\begin{array}{l}\text { The server broadcasts a message to the entire network and each anchor node also broad- } \\
\text { casts a message to the entire network }\end{array}$ \\
\hline DV-RND & $\begin{array}{l}\text { Each neighbouring node obtains its information through the transmission and forwarding } \\
\text { by node i, and then each anchor node broadcast two messages to the entire network. }\end{array}$ \\
\hline
\end{tabular}


Table 1 shows that the LEAP has the smallest traffic volume, followed in ascending order by DV-HOP, DV-RND and PNN-MAP. The PNN-MAP surpassed the other three algorithms, because it has to transmit the node information to the server. However, the nodes in PNN-MAP are responsible for information transfer only. The calculations are done in the server, rather than the nodes. Through the calculation, the server can learn about the positions of the nodes. In all the other algorithms, the nodes must have a certain calculation ability, and transfer the calculated results to the server. All these push up the traffic volume. Through the above analysis, the PNN-MAP and the other three algorithms have different characteristics in energy consumption and should be selected as per specific requirements. However, the PNN-MAP has an obvious edge over the other algorithms in EDE and LE.

\section{Conclusions}

In WSNs, the node positioning is essential to the robustness of the data collected by each node, and the key to the application of the entire network. In this paper, the PNNMAP algorithm, a proximity-based range-free positioning approach, is presented to overcome the large EDE and LE of hop-based range-free positioning algorithms.

First, the proximity was introduced to express the distance between neighbouring nodes and the proximity expression was derived based on the geometric relationship between these nodes. Then, the estimated distance was calculated by the proximity expression and relevant information of anchor nodes, and the unknown node positions were estimated by the MDS-MAP to reduce the LE. Later, the PNN-MAP algorithm was simulated and compared to three other node positioning algorithms. Through the simulation, this algorithm was proved effective as it achieved the smallest EDE and LE at different communication radiuses or different number of anchor nodes. In terms of energy consumption, the PNN-MAP had a relatively high traffic volume. However, there is no requirement on the calculation ability of network nodes, and the estimated node positions can be displayed directly on the server after the positioning. In general, the PNN-MAP enjoys a strong practicality and universality.

\section{$5 \quad$ References}

[1] Agrawal, N., Milner, S.D., Davis, C.C. (2014). Design and performance of a directional media access control protocol for optical wireless sensor networks. IEEE/OSA Jour-nal of Optical Communications \& Networking, 6(2): 215-224. https://doi.org/10.1364/JOCN. $\underline{6.000215}$

[2] Kim, Y.D., Kang, W.S., Cho, K., Kim, D. (2013). Rmrp: a reliable mac and routing protocol for congestion in IEEE 802.15.4 based wireless sensor networks. IEICE Trans-actions on Communications, 96(12): 2998-3006. https://doi.org/10.1587/transcom.E96.B.2998

[3] Chen, H., Fan, G., Xie, L., Cui, J.H. (2013). A hybrid path-oriented code assignment cdmabased mac protocol for underwater acoustic sensor networks. Sensors, 13(11): 15006-15025. https://doi.org/10.3390/s131115006 
[4] Li, M., Li, Z., Vasilakos, A.V. (2013). A survey on topology control in wireless sensor networks: taxonomy, comparative study, and open issues. Proceedings of the IEEE, 101(12): 2538-2557. https://doi.org/10.1109/JPROC.2013.2257631

[5] Han, G., Jiang, J., Shu, L., Xu, Y., Wang, F. (2012). Localization algorithms of under-water wireless sensor networks: a survey. Sensors,12(2): 2026-2061. https://doi.org/10.3390/ $\underline{\text { s120202026 }}$

[6] Niewiadomska-Szynkiewicz, E., Marks, M. (2009). Optimization schemes for wireless sensor network localization. International Journal of Applied Mathematics \& Com-puter Science, 19(2): 291-302. https://doi.org/10.2478/v10006-009-0025-3

[7] Yildirim, K. S., Kantarci, A. (2013). Time synchronization based on slow-flooding in wireless sensor networks. IEEE Transactions on Parallel \& Distributed Systems, 25(1): 244-253. https://doi.org/10.1109/TPDS.2013.40

[8] Yang, X., Tao, X., Dutkiewicz, E., Huang, X., Guo, Y. J., Cui, Q. (2013). Energy-efficient distributed data storage for wireless sensor networks based on compressed sensing and network coding. IEEE Transactions on Wireless Communications, 12(10): 5087-5099. https://doi.org/10.1109/TWC.2013.090313.121804

[9] He, D., Chen, C., Chan, S., Bu, J., Yang, L. T. (2013). Security analysis and improve-ment of a secure and distributed reprogramming protocol for wireless sensor net-works. IEEE Transactions on Industrial Electronics, 60(11): 5348-5354. https://doi.org/10.1109/TIE. 2012.2218562

[10] Chen, T., Yang, Z., Liu, Y., Guo, D., Luo, X. (2013). Localization-oriented network adjustment in wireless ad hoc and sensor networks. IEEE Transactions on Parallel \& Distributed Systems, 25(1): 146-155. https://doi.org/10.1109/TPDS.2013.17

[11] Hou, C., Hou, Y., Huang, Z. (2013). A framework based on barycentric coordinates for localization in wireless sensor networks. Computer Networks, 57(17): 3701-3712. https://doi.org/10.1016/j.comnet.2013.08.014

[12] Kim, W., Park, J., Yoo, J., Kim, H. J., Chan, G. P. (2013). Target localization using ensemble support vector regression in wireless sensor networks. IEEE Transactions on Cybernetics, 43(4): 1189-1198. https://doi.org/10.1109/TSMCB.2012.2226151

[13] Patwari, N., Ash, J. N., Kyperountas, S., Hero, A. O. I., Moses, R. L., Correal, N. S. (2005). Locating the nodes: cooperative localization in wireless sensor networks. IEEE Signal Processing Magazine, 22(4): 54-69. https://doi.org/10.1109/MSP.2005.1458287

[14] Hadjidj, A., Souil, M., Bouabdallah, A., Challal, Y., Owen, H. (2013). Wireless sensor networks for rehabilitation applications: challenges and opportunities. Journal of Network \& Computer Applications, 36(1): 1-15. https://doi.org/10.1016/j.jnca.2012.10.002

[15] Chiara, B., Andrea, C., Davide, D., Roberto, V. (2009). An overview on wireless sensor networks technology and evolution. Sensors, 9(9): 6869-96. https://doi.org/10.3390/s9090 $\underline{6869}$

\section{Author}

Huaimeng Gui was born in Shaanxi, China in 1985. She received the Doctor degree from Xi'an university of technology, China in 2015. She is currently a Lecturer with Shaanxi Polytechnic Institute, Shaanxi, China. Her research interests include electron device and application.

Article submitted 30 July 2018. Resubmitted 05 September 2018. Final acceptance 09 October 2018. Final version published as submitted by the author. 